\title{
PERMANENT CHANGES IN SPORADIC E LAYERS OVER FORTALEZA, BRAZIL
}

\author{
M. A. Abdu*, I. S. Batista*, J. MacDougall**, J. H. A. Sobral* and P. Muralikrishna* \\ *Instituto Nacional de Pesquisas Espaciais, INPE, C.P. 515, 12201-970, São José \\ dos Campos, SP, Brazil \\ **Department of Engineering, University of Western Ontario, London, N6 A5 B9, Canada
}

\begin{abstract}
Data available on sporadic E layers in ionograms, from 1975 to 1995 over Fortaleza $\left(38^{\circ} \mathrm{W}, 4^{\circ} \mathrm{S}\right)$ in Brazil are analysed as an extension of a prcvious study to examine permanent changes in the occurrence characteristics of the different types of $\mathrm{E}_{\mathrm{s}}$ layers. The so called $\mathrm{q}$ type $\mathrm{E}_{\mathrm{s}}$ produced by plasma instability (type II) process of the equatorial electrojet and the other types of $\mathrm{E}_{s}$ produced by wind/wind shear mechanism are studied separately. The results show that while the occurrence of the former variety steadily decreased during the period of analysis, the latter type showed marked increases. These characteristics are shown to be associated mainly with the steady drift of the magnetic equator to northward of Fortaleza at a rate of $\sim 0.2$ degree per year. The long term trends are, however, modulated by solar activity cycle in $F_{10.7}$ flux. Also there is a suggestion of a possible effect from the June 1991 Pinatubo volcanic eruption. The competing roles of the neutral winds and electric fields in the formation of the low latitude sporadic $E$ layer is demonstrated.

(C1997 COSPAR. Published by Elsevier Science Ltd.
\end{abstract}

\section{INTRODUCTION}

There is an increasing interest in the scientific community to understand the permanent or long term changes that take place in the upper layers of the earth's atmosphere. It has been found that the ionosphere-thermosphere-mesosphere system presents better sensitivity to certain aspects of the long term changes than does the lower atmosphere (Rishbeth, 1996, this issue). Such changes could occur from natural such as solar-geophysical processes, secular changes in the earth's interior source of the geomagnetic field, volcanic eruptions, etc., or by anthropogenic sources. Reliable long term observational data base is necessary for investigating such changes. Further the task of identifying an observed change as arising from, say, anthropogenic activities requires detailed assessment of all other possible contributions from natural sources of such changes, and vice versa. In this paper we have focussed attention on aspects of long term changes arising from natural sources. We have examined the long term trend in the sporadic E-layer phenomenon over an equatorial station, Fortaleza, in Brazil using a 21-year (1975-1995) data base. This work which is an extension of a recent study published by Abdu et al. (1996), that covered only the period 1975-1990, shows that significant changes of permanent nature have taken place for the sporadic $E$ layer development conditions over Fortaleza. Such changes arise mainly from natural processes as will be explained in this paper.

\section{SPORADIC E-LAYER DATA SET}

Different types of $E_{s}$ layers occur in the equatorial electrojet and its low latitude vicinity regions. The q-type layer observed at $105-110 \mathrm{~km}$ in the day time equatorial ionograms is a manifestation of the type II irregularities of the equatorial electrojet (EE.) produced by the well-known gradient E x B instability mechanism (Reid, 1960) that is primarily driven by the eastward global $E$ region dynamo electric field. Other types of $E_{s}$ layers, $1, f, h$, and $c$ types (see URSI Handbook of Ionogram Interpretation and Reduction, second edition, 1972, Report UAG-2) that occur in the low latitude regions outside of the EEJ are produced by wind/wind shear mechanism (see, Axford, 1963; Whitehead, 1961; Abdu and Batista, 1977). The 1 and $f$ types (hereafter referred to as $1 / f$ type) occur during the day and night respectively. The h-type develops around $150 \mathrm{~km}$ and descending in sequential ionograms (MacDougall, 1974; Wilkinson et al., 
1992) becomes c-type $E_{s}$. We therefore refer them as $c / h$ type. The statistical analysis on the occurrences of the different types of $E_{s}$ are presented here for the September month of the years from 1975 to 1995.

\section{RESULTS}

Figure 1 presents, as a function of local time, the hourly occurrence percentages, with respect to the total number of soundings carried out in the month of September, of the years: 1975-77, 1979-80, 1982-84, 1986-87, 1989-92, and 199495. The following features may be noted: (1) The $q$-type $E_{s}$ is restricted to daytime only. In the earlier years starting from 1975 its occurrence rises sharply after sunrise and reaches highest values approaching $\sim 100$ percent before noon and decreases to zero value around sunset. There is an afternoon enhancement that gets dominant during the years 19771980 centered around solar maximum; (2) The q-type $E_{s}$ occurrence rate decreases steadily from 1982 (with $\sim 90$ percent) to negligibly small value in 1989 (with 5-10 percent); it shows slight increase (up to 20 percent) in 1990 but almost total disappearance in 1991 and then a tendency for a small increase confined to afternoon hours during the years 1992-95; (3) The $\mathrm{h} / \mathrm{c}$ type events occur only during sunlit hours with two maxima, one in the morning and the other in the evening. The amplitude of these maxima increases from 20 percent in 1975 to $\sim 60$ percent in 1990. Then there is a total disappearance of this phenomenon in 1991 followed by a minor recovery during the years 1992-95; (4) The 1/f-type $E_{s}$ that occurs generally in the $100-105 \mathrm{~km}$ region has higher occurrence rate during the night than during the day, a feature that is present till 1990 (with a possible exception in 1989). Starting from 1991 its diurnal variation does not present any consistent pattern. Its daytime occurrence rate showed striking increase to $\sim 50$ percent in 1990 from almost negligible occurrence in 1975. Later the peak occurrence (in an altered diurnal pattern) remained nearly steady till 1994 and then decreased to small values in 1995.

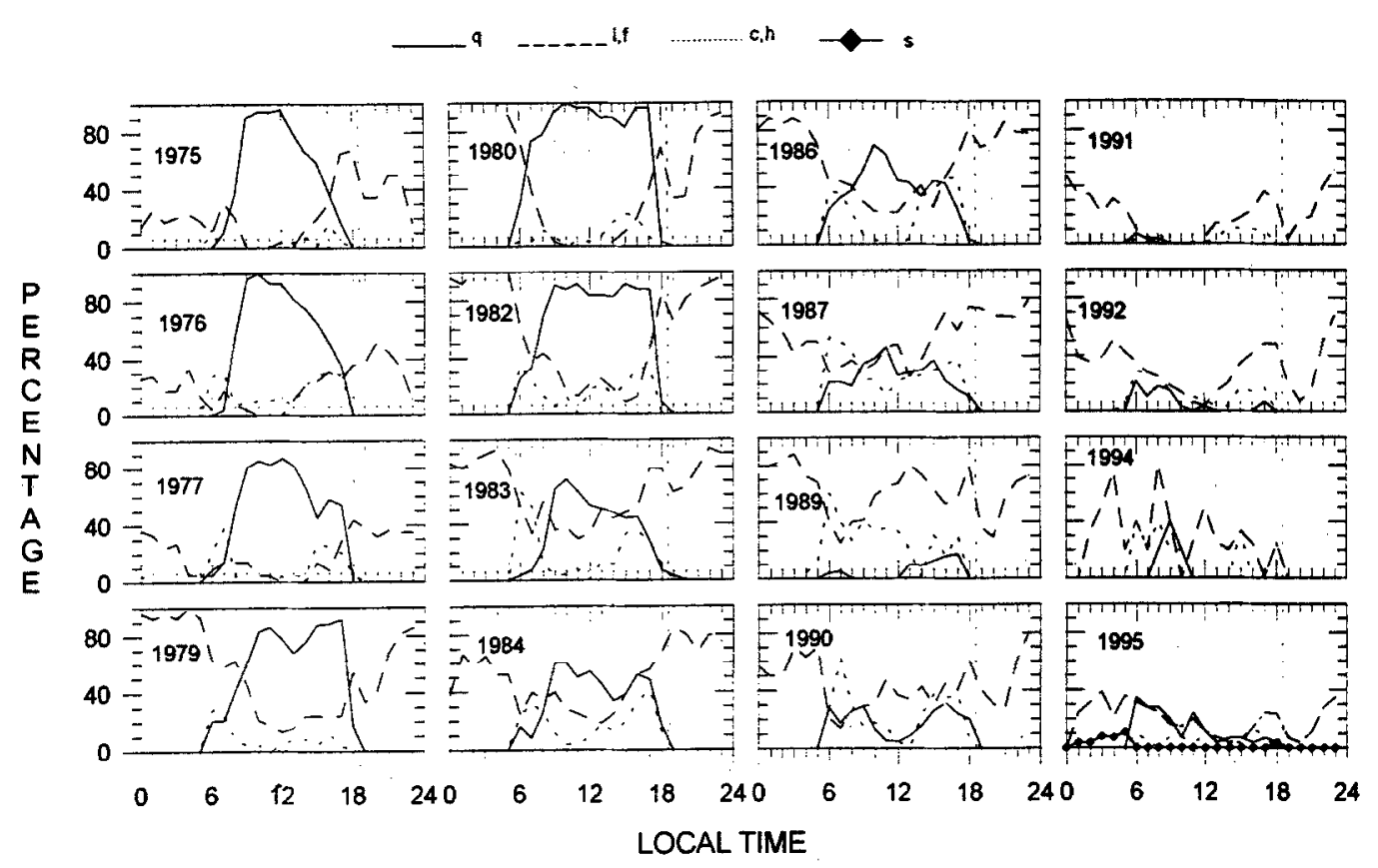

Fig. 1. Diurnal patterns of the $\mathrm{q}, \mathrm{l} / \mathrm{f}, \mathrm{h} / \mathrm{c}$ types of $\mathrm{E}_{\mathrm{s}}$ hourly percentage occurrences for the September months of years from 1975 to 1995 , over Fortaleza.

The $\mathrm{E}_{s}$ occurrence rates averaged for the hours 1000-1400 LT are presented in the middle panel of Figure 2 as a function of years from 1975 to 1995 . Shown in the bottom panel is the yearly average solar $F_{10.7}$ flux. The top panel shows the variation of distance of Fortaleza station from the magnetic equator as per the International Geomagnetic Reference Field (IGRF) model. This figure permits us to analyse the long term changes in the different types of $\mathrm{E}_{\mathrm{s}}$ layers as a function of the increasing distance of the station from the EEJ during the 21-years period as well as a function of solar activity cycle. We may note the following important characteristics: (1) The q-type $\mathrm{E}_{\mathrm{s}}$ decreased from $\sim 90$ percent 


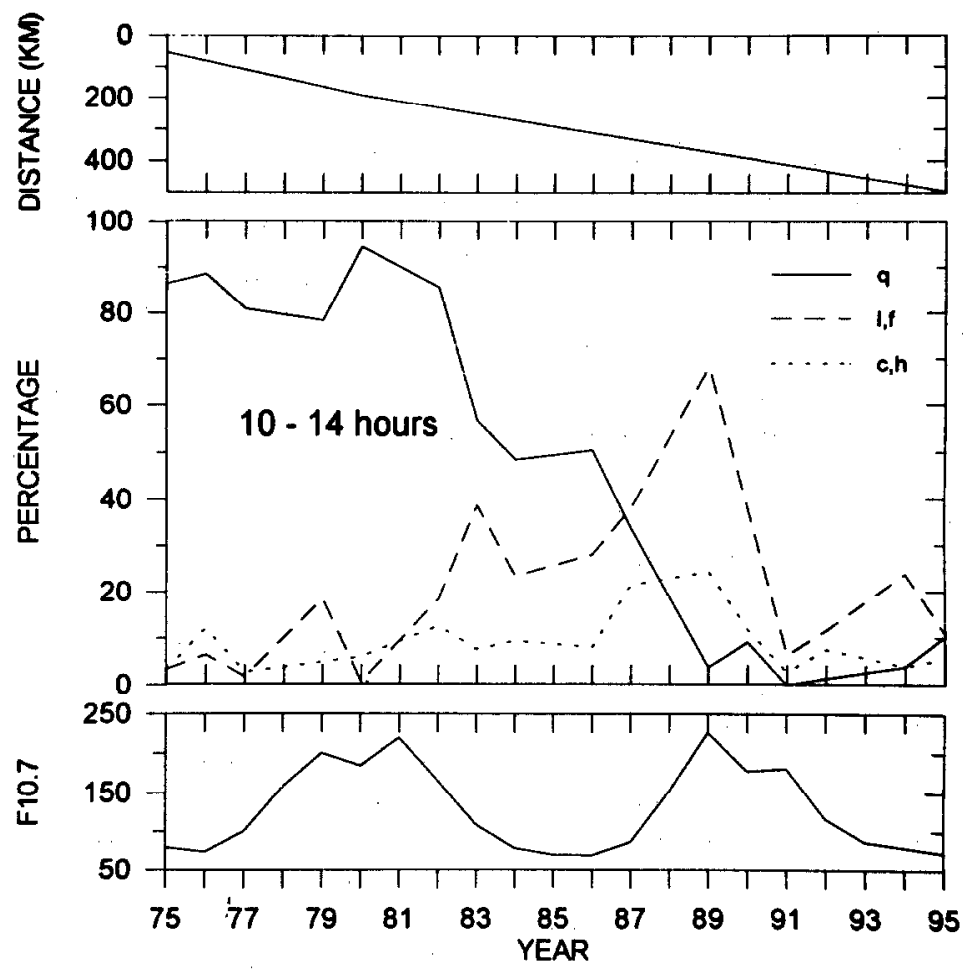

Fig. 2. Top panel : The distance of Fortaleza station from the magnetic equator (in km) from 1975 to 1995; Middle panel : Percentage occurrences of the $\mathrm{q}, \mathrm{l} / \mathrm{f}, \mathrm{h} / \mathrm{c}$ types of the $\mathrm{E}_{\mathrm{s}}$ layers over Fortaleza as a function of the years from 1975 to 1995 ; Bottom panel: The $F_{10.7}$ solar radio flux for the period 1975-1990.

occurrence in 1975 to almost insignificant value around 1989 when the magnetic equator, in its secular drift reached some $400 \mathrm{~km}$ north of Fortaleza. Its occurrence remained at this low rate till 1995. This indicates a permanent change in the q-type $\mathrm{E}_{s}$ occurrence phenomena over Fortaleza brought about by the displacement of the electrojet away from this station. The $1 / f$ and $h / c$ types of $E_{s}$ showed significant enhancements towards 1989 suggesting that their occurrences increased with increasing distance of Fortaleza from the EEJ center. Their occurrences decrease after 1989; (2) There are modulation cycles superimposed over the long term trend; that are often in opposite phases in the q-type on one hand and in the $1 / f$ and $\mathrm{h} / \mathrm{c}$ types on the other. These modulations seem to be controlled by the solar flux values. For example, the broad maximum centered around 1980 with respect to an othcrwise decreasing trend, in the q-type $\mathrm{E}_{\mathrm{s}}$, appears to be produced by processes associated with the solar cycle maximum in the $F_{10.7}$ flux. However, the $F_{10.7}$ maximum of 1989 did not cause any enhancement in the q-type $E_{s}$ which is understandable by the fact that its formation is mainly controlled by the electric field of the EEJ which has moved away from Fortaleza. In contrast the $1 / f$ and $h / c$ types of $E_{s}$ showed maxima corresponding to the maximum in $F_{10.7}$; (3) The occurrence rate presents very small value in 1991. This is significantly lower than expected for the $F_{10.7}$ value though it was on a declining trend from its solar cycle maximum of 1989. In the absence of any other identifiable cause we are tempted to attribute this strikingly small occurrence of the $E_{s}$ layers in September 1991 to possible effect of the Pinatubo volcanic eruption that occurred in the Philippines $\left(15^{\circ} \mathrm{N}, 120^{\circ} \mathrm{E}\right)$ on 15-16 June 1991. The propagation of the aerosol clouds to the longitude of Brazil took around two weeks as verified from lidar observations in São José dos Campos (Dale Simonich, Private communication). However, the validity of this speculation needs to be verified from detailed analysis of the available data sets. Over all, while Figure 2 presents some permanent changes in the sporadic $E$ layer phenomenon over Fortaleza it seems to present also solar cycle effects in their driving forces, that is, electric fields and winds. It seems to show further a possible effect of a major volcanic eruption. 


\section{JISCUSSION AND CONCLUSIONS}

is far as Fortaleza is concerned the results of analysis of the sporadic $E$ layer phenomenon shows permanent changes vith regard to the occurrences of all types of $\mathrm{E}_{s}$ layers. However, we should keep in mind that these changes are brought bout by the secular drift of the magnetic equator to northward of that station. The consequent implications of the elative roles of electric field and neutral winds on the $\mathrm{E}_{s}$ layer formation as a function of distance from the EEJ center las been discussed in Abdu et al. (1996). In that paper the results were presented based on the data up to 1990. The xtension of the analysis up to 1995 carried out in this paper has permitted a study of the $\mathrm{E}_{\mathrm{s}}$ layer formation for about wo solar activity cycles. The maxima of the first of these, centered around 1980 did produce significant increase in he q-type $\mathrm{E}_{\mathrm{s}}$ layer occurrence. This signifies a corresponding increase in the Hall polarization electric field in the EEJ hat is driven by the E-layer dynamo eastward electric field. The equatorward daytime electric field has been shown o have little dependence on solar activity cycle (Fejer et al., 1991). Therefore the q-type $E_{s}$ layer occurrence dependence in $F_{10.7}$ flux in Figure 2 signifies a solar activity effect on the Hall electric field brought about by corresponding changes n the collision frequency and electron density in the F-region. Such a dependence is reinforced by the occurrence of maximum in the $1 / f$ and $h / c$ types of $E_{s}$ (that is, the $E_{s}$ layers produced by wind/wind shear mechanism) in association vith the $F_{10.7}$ maximum of the solar cycle peak of 1989 . The ion convergence process that form $E_{s}$ layers depend upon a) the ratio of ion-neutral collision frequency to ion gyrofrequency, and (b) on the magnitude of the vertical shears in he zonal wind or just on the magnitude of a zonal wind (Abdu and Batista, 1977). Therefore the above correlation ignifies also a solar cycle dependence of the collision frequency and wind/wind shear magnitudes. An intriguing aspect if the present results is the observation of negligible occurrence of daytime wind induced $\mathrm{E}_{\mathrm{s}}$ layer over Fortaleza during he September month of 1991 (for which analysis was done here) which appears to show a recovery trend in the later one or two years. The possible cause of such a behaviour is under investigation. In conclusion we may note that: (1) rermanent changes in the occurrence rates of different types of $E_{s}$ layers have occurred over Fortaleza since 1975 rrought about by the secular drift of the magnetic equator to northward of that station, and (2) the long term trend that ;overn these changes could be modulated by solar activity effects and perhaps also other natural processes such as major olcanic eruptions.

\section{ICKNOWLEDGEMENTS}

The authors wish to acknowledge the support from the Conselho Nacional de Desenvolvimento Cientifico e ecnológico-CNPq through grants $\mathrm{n}^{\mathrm{o}}$ 520185/95-1 and 521980/94-1.

\section{LEFERENCES}

Ibdu, M. A., and I. S. Batista, Sporadic E-layer Phenomena in the Brazilian Geomagnetic Anomaly: Evidence for a legular Particle Ionization Source. J. Atmos. Terr. Phys., 39, 723-731 (1977).

tbdu, M. A., I. S. Batista, P. Muralikrishna, and J. H. A. Sobral, Long Term Trends in Sporadic E Layers and Electric iields Over Fortaleza, Brasil. Geophys. Res. Lett., 23, 757-761 (1996).

txford, W. I., The Formation and Vertical Movements of Dense Ionized Layers in the Ionosphere Due to Neutral Wind ihears. J. Geophys. Res., 68, 769-779 (1963).

ejer, B. G., E. R. de Paula, S. A. Gonzales, and R. F. Woodman, Average Vertical and Zonal F Region Plasma Drifts Jver Jicamarca. J. Geophys. Res., 96, 13901-13906 (1991).

MacDougall, J. W. 110 km Neutral Zonal Wind Patterns. Planet. Space Sci., 22, 545-558 (1974).

Reid, G. C., The Formation of Small Scale Irregularities in the Ionosphere, J. Geophys. Res., 73, 1627-1640 (1968). lishbeth, H., Long Term Changes in the Ionosphere. XXXI COSPAR, Birmingham, England (1996).

Nhitehead, J. D., The Formation of the Sporadic- E Layer in the Temperate Zone. J. Atmos. Terr. Phys., 20, 49-58 1961).

Nilkinson, P. J., E. P. Szuszczewicz, and R. G. Roble, Measurements and Modelling of Intermediate, Descending, and 'poradic Layers in the Lower Ionosphere: Results and Implications for Global-scale Ionospheric - Thermospheric itudies. Geophys. Res. Letts., 19, $95-98$ (1992). 